\title{
Coevolutionary phage training leads to greater bacterial suppression and delays the evolution of phage resistance
}

\author{
Joshua M. Borin ${ }^{1}$, Sarit Avrani ${ }^{2}$, Jeffrey E. Barrick ${ }^{3}$, Katherine L. Petrie ${ }^{1,4}$, and Justin R. \\ $\operatorname{Meyer}^{1 *}$ \\ 1. Division of Biological Sciences, University of California San Diego 92093 \\ 2. Department of Evolutionary and Environmental Biology and The Institute of Evolution, University of Haifa \\ 3498838 \\ 3. Department of Molecular Biosciences, The University of Texas at Austin 78712 \\ 4. Earth-Life Science Institute, Tokyo Institute of Technology, Tokyo, Japan 145-0061 \\ *corresponding author \\ Classification \\ BIOLOGICAL SCIENCES, Evolution
}

Keywords

Evolution, Coevolution, Resistance, Phage therapy, Phage training

\section{Author Contributions}

JMB, JRM, and SA designed research; JMB, KLP and JRM performed research; JMB and JRM analyzed data; JMB and JRM wrote the paper; SA and JRM provided financial support. All authors edited the manuscript.

\section{Significance Statement}

The evolution of antibiotic resistant bacteria threatens to claim over 10 million lives annually by 2050. This crisis has renewed interest in phage therapy, the use of bacterial viruses to treat infections. A major barrier to successful phage therapy is that bacteria readily evolve phage resistance. One idea proposed to combat resistance is "training" phages by using their natural capacity to evolve to counter resistance. Here, we show that training phages by coevolving them with their host for one month enhanced their capacity for suppressing bacterial growth and delayed the emergence of resistance. Enhanced suppression was caused by several mechanisms, suggesting that the coevolutionary training protocol produces a robust therapeutic that employs complementary modes of action. 


\begin{abstract}
The evolution of antibiotic resistant bacteria threatens to become the leading cause of worldwide mortality. This crisis has renewed interest in the practice of phage therapy. Yet, bacteria's capacity to evolve resistance is likely to debilitate this therapy as well. To combat the evolution of phage resistance and improve treatment outcomes, many have suggested leveraging phages' ability to counter resistance by evolving phages on target hosts before using them in therapy (phage training). We found that during in vitro experiments, a phage trained for 28 days suppressed bacteria 1000-fold for 3-8 times longer than its untrained ancestor. This extension was due to a delay in the evolution of resistance. Several factors contributed to this prolonged suppression. Mutations that confer resistance to trained phages are $\sim 100 \times$ less common and, while the target bacterium can evolve complete resistance to the untrained phage in a single step, multiple mutations are required to evolve complete resistance to trained phages. Mutations that confer resistance to trained phages are more costly than mutations for untrained phage resistance. And when resistance does evolve, trained phages are better able to suppress these forms of resistance. One way the trained phage improved was through recombination with a gene in a defunct prophage in the host genome, which doubled phage fitness. This direct transfer of information encoded by the host but originating from a relict phage provides a previously unconsidered mode of training phage. Overall, we provide a case study for successful phage training and uncover mechanisms underlying its efficacy.
\end{abstract}




\section{Introduction}

In 30 years, the World Health Organization predicts that antibiotic resistant bacteria will kill over 10 million people each year-more deaths than that caused by cancer (1). This health crisis, in part caused by the heavy and often inappropriate way we use antibiotic drugs, has led to the spread of resistance genes through clinical and natural environments and to the emergence of multidrug resistant (MDR) "superbugs" that are often untreatable due to their resistance against all available classes of antibiotics (2-4). As bacteria continue to outpace our discovery and development of new drugs, the evolution of resistance threatens to return us to a pre-antibiotic era of infectious disease $(5,6)$.

This crisis has renewed interest in the century-old practice of phage therapy: the use of phages, viruses that infect bacteria, to treat bacterial infections (7-11). Recently, phage therapy has shown promise in cases where drugs of last resort fail to treat life-threatening MDR bacterial infections (9-13). However, even in successful cases, the evolution of phage resistance poses a considerable threat to the efficacy of treatment $(9,12,14)$. For example, in 2016 , at the University of California San Diego, a patient with acute pancreatitis complicated by an MDR Acinetobacter baumanii infection was treated with two 4-phage cocktails that suppressed the pathogen in vitro (9). Within 8 days, A. baumanii isolated from the patient was resistant to all 8 phages used. Fortunately, the infection resolved following delivery of a ninth phage and the patient survived. This case is representative of numerous phage therapy studies (9-12,14). A metanalysis in 2018 reported that phage resistance evolved in $82 \%$ of animal gut decolonization studies, $50 \%$ of meningitis/sepsis models, and $75 \%$ of human clinical cases where the evolution of resistance was monitored (14). These observations of rapid phage resistance evolution in therapy mirror decades of basic research in the lab; mutations that confer resistance to phages are often as common as those for antibiotic resistance (15-17). Furthermore, many of these resistance mutations confer cross-resistance to multiple phages (18).

Although resistance to phages is as or more common than to antibiotics, advantages of using phages as therapeutics have been proposed time and again $(7,8,19-22)$. Notably, unlike antibiotics, phages are biological entities that evolve. By reciprocally adapting to changes in their hosts (coevolution), phages have maintained the ability to infect their hosts for millennia. Many have proposed harnessing this inherent, evolutionary potential by preemptively coevolving phages with target bacterial prey (22-24). Proponents of this "phage training" approach suggest that, by experiencing the ways their host can evolve resistance, trained phages will evolve to counter host defenses. Then, trained phages "from the future" can be used to trap the ancestral, un-coevolved bacteria from their past that are infecting the patient.

While the idea of phage training is enticing, it has not yet been adopted for therapy. Contrasting theories of bacteria-phage coevolutionary dynamics make the success of phage training uncertain $(12,24,25)$. According to some conceptual models of coevolution (e.g. matching alleles), as phages adapt to their evolving host, they lose the ability to infect past hosts (24). In such cases, phage training would not work because trained phages would lose the ability to infect the original target bacterium. Alternatively, other models of coevolution (e.g. gene for gene, arms race dynamics) argue that as phages adapt to their evolving host, they maintain the ability to infect their original host (24). In this scenario, training would expand phage host range to encompass both original and contemporary bacteria. Regardless of how coevolution affects host 
range, some opponents of phage training contend that the use of trained phages will apply stronger selection on target bacteria which will accelerate the evolution of resistance and loss of therapeutic efficacy (22).

In this study, we conducted a coevolution experiment using Escherichia coli and either untrained or trained phages to evaluate the potential of phage training for therapy. By comparing the population dynamics of coevolving bacteria and phages, we find that trained phages suppress the target bacteria more strongly and for longer than untrained phages. Through post hoc analyses on the bacteria and phages that evolved in our experiment, we identify the factors that allowed trained phages to suppress host populations and delay the evolution of resistance.

\section{Results}

Coevolution Experiment. We propagated populations of E. coli B strain REL606 with either untrained or trained strains of phage $\lambda$ in flasks for 30 days and compared the population dynamics of coevolving bacteria and phages. For untrained phages, we used $\lambda$ unt, a lytic strain of $\lambda$ that uses LamB as a receptor. For trained phages, we used $\lambda$ trn, a descendant of $\lambda$ unt that was isolated on day 28 of a previous coevolution experiment with REL606 and that can infect using either of two receptors, LamB and $\mathrm{OmpF}(26,35)$. Because $\lambda$ trn uses two receptors, we hypothesized that it would be more difficult for hosts to evolve envelope-based resistance.

Daily measurements of population densities revealed large differences in bacterial abundance between treatments (Fig. 1A), whereas phage densities were similar (Fig. 1B). By day 3, bacteria coevolving with $\lambda$ unt were no longer suppressed by phages and were instead limited by the availability of glucose in the media. This rapid loss of suppression is consistent with past studies on $\lambda$ unt and REL606 (26-28), as well as studies on other bacteria-phage pairs (29,30); when cultured with phages in flasks, bacteria rapidly evolve resistance and their growth is limited by the availability of nutrients. However, in flasks with $\lambda$ trn, phages suppressed their hosts for 1328 days, at which point bacterial densities slowly increased until they became limited by the availability of glucose, as in flasks with $\lambda$ unt. The prolonged suppression of bacteria by $\lambda \operatorname{trn}$ suggested that resistance to $\lambda$ trn evolved much later than to $\lambda$ unt. 

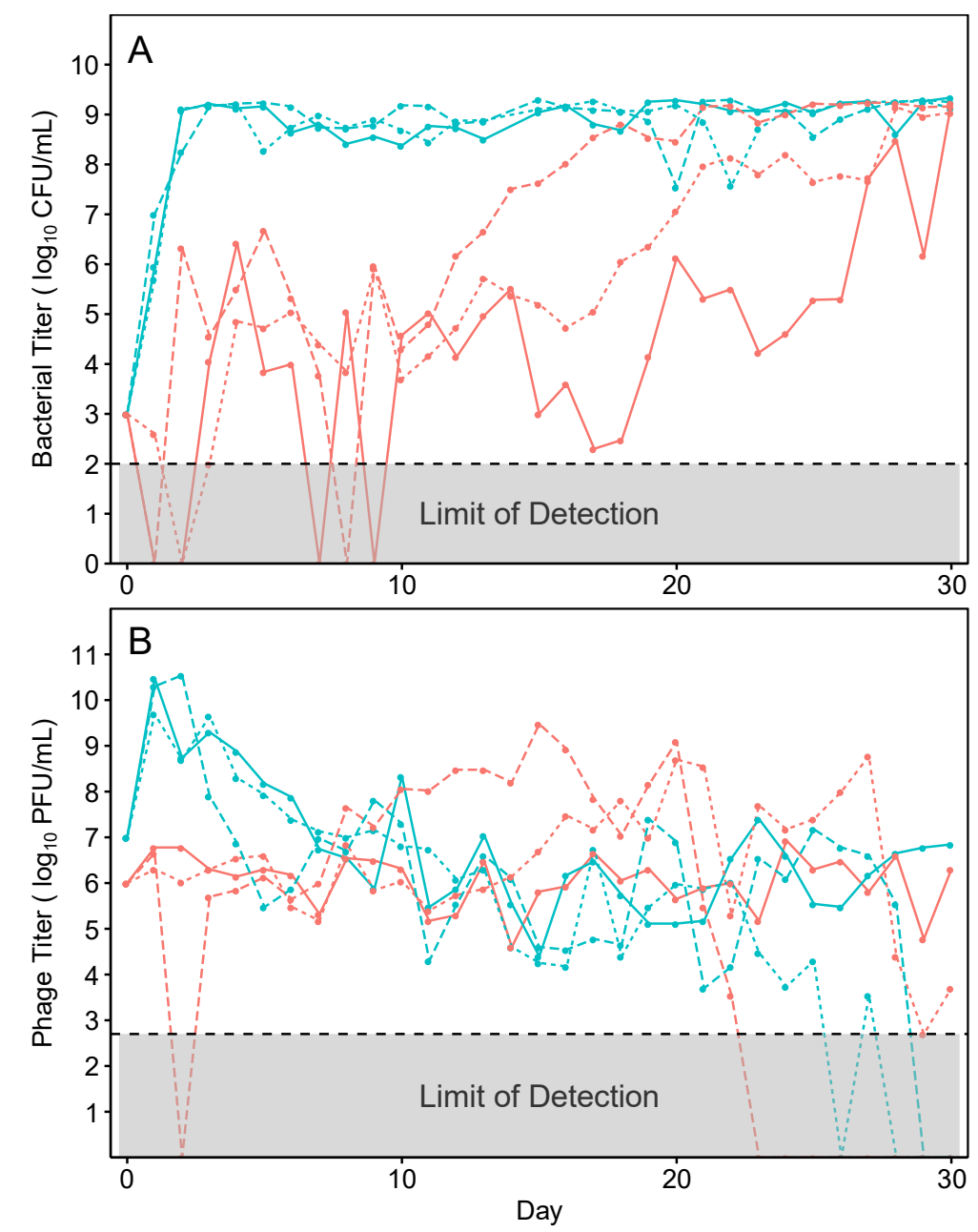

Figure 1. Population dynamics of bacteria (A) and phage (B) during 30 days of coevolution in flasks, estimated from colony forming units (CFU) and plaque forming units (PFU), respectively. Flasks containing $\lambda$ unt are teal and $\lambda$ trn are red. Line types correspond with replicate Populations ( $\operatorname{solid}=1$, dotted=2, dashed=3).

Emergence of Resistance. To determine when resistance evolved in each treatment, we sampled bacteria from various timepoints and challenged them with the initial phage they were cocultured with ( $\lambda$ unt or $\lambda$ trn). We assessed the resistance of 12 isolates every 5 days to quantify the diversity of resistance. Initially, we performed spot assays where $5 \mu \mathrm{L}$ of phage lysate was spotted on top of an isogenic lawn of bacteria growing on an agar plate. We found that this approach was inadequate because many bacterial isolates appeared sensitive to $\lambda$ trn when grown on agar (clearing due to phage killing) but were able to grow with phage in liquid culture.

Given that the experiments were conducted in liquid medium, we developed a protocol to efficiently track bacterial population growth in liquid culture using a microtiter plate reader. Resistance was categorized visually by comparing the difference in cell density (via optical density; OD) dynamics between cultures growing with and without phage. Bacteria were deemed sensitive if no growth was observed in the presence of phage and completely resistant if cells grew uninhibited by phage. Partial resistance was recorded for bacteria that showed signs of growth but were clearly inhibited by the phage (representative isolates in Fig. S1). 
Using the liquid-based assay described above, we characterized the proportion of resistance in populations of the coevolution experiment at various time-points (Fig. 2, A-F). Resistance to $\lambda$ unt evolved much earlier than to $\lambda$ trn; by day $3, \geq 50 \%$ of isolates were partially or completely resistant to $\lambda$ unt and by day $10,100 \%$ of isolates were completely resistant. Despite high levels of resistance, $\lambda$ unt was able to persist. This was likely due to genetically "leaky resistance", in which a resistant population of bacteria continuously generates a small number of sensitive mutant cells upon which a phage population can subsist (31). As in previous coevolution experiments, $\lambda$ unt in populations 1, 2, and 3 evolved to use OmpF as a receptor on days 17, 22, and 19 , respectively.

In contrast to flasks with $\lambda$ unt, the evolution of partial and complete resistance to $\lambda$ trn occurred later and was more variable. In $\lambda$ trn Population 3, partial resistance evolved by day 5 but complete resistance was not detected until day 15 (Fig. 2F). However, phages isolated 5 days later were able to infect these completely resistant isolates (Fig. S2). In $\lambda$ trn Population 2, partial resistance was not detected until day 15 . One completely resistant strain was isolated from day 25 but it was not detected at later time-points. Phages isolated two days later were able to infect this completely resistant isolate (Fig. S2). These results suggest that $\lambda$ trn's ability to evolve counter-defenses may have contributed to the disappearance of the resistant genotype. Lastly, in $\lambda$ trn Population 1, partial resistance was not detected until day 25 and complete resistance never evolved (Fig. 2D). Surprisingly, even at the end of the experiment, day-30 isolates from $\lambda$ trn Population 1 lacked resistance, as determined by spot and liquid-based assays (Fig. 2D). We further describe this case in SI Appendix: $\lambda$ trn Resistance.

Delay in the evolution of resistance against our trained phage explains the difference in suppression between flasks containing $\lambda$ unt and $\lambda$ trn. To understand the reasons for this delay, we investigated four non-mutually exclusive hypotheses. First, $\lambda$ trn resistance would take longer to evolve if there were fewer mutations available that confer resistance to this phage. Second, resistance would be delayed if more mutations were required to confer resistance to $\lambda$ trn than $\lambda$ unt. Third, if mutations for $\lambda$ trn resistance are more costly, it will take them longer to rise in frequency. And fourth, $\lambda$ trn may be better able to evolve counter-defenses, which would prolong its ability to suppress bacterial resistance. 

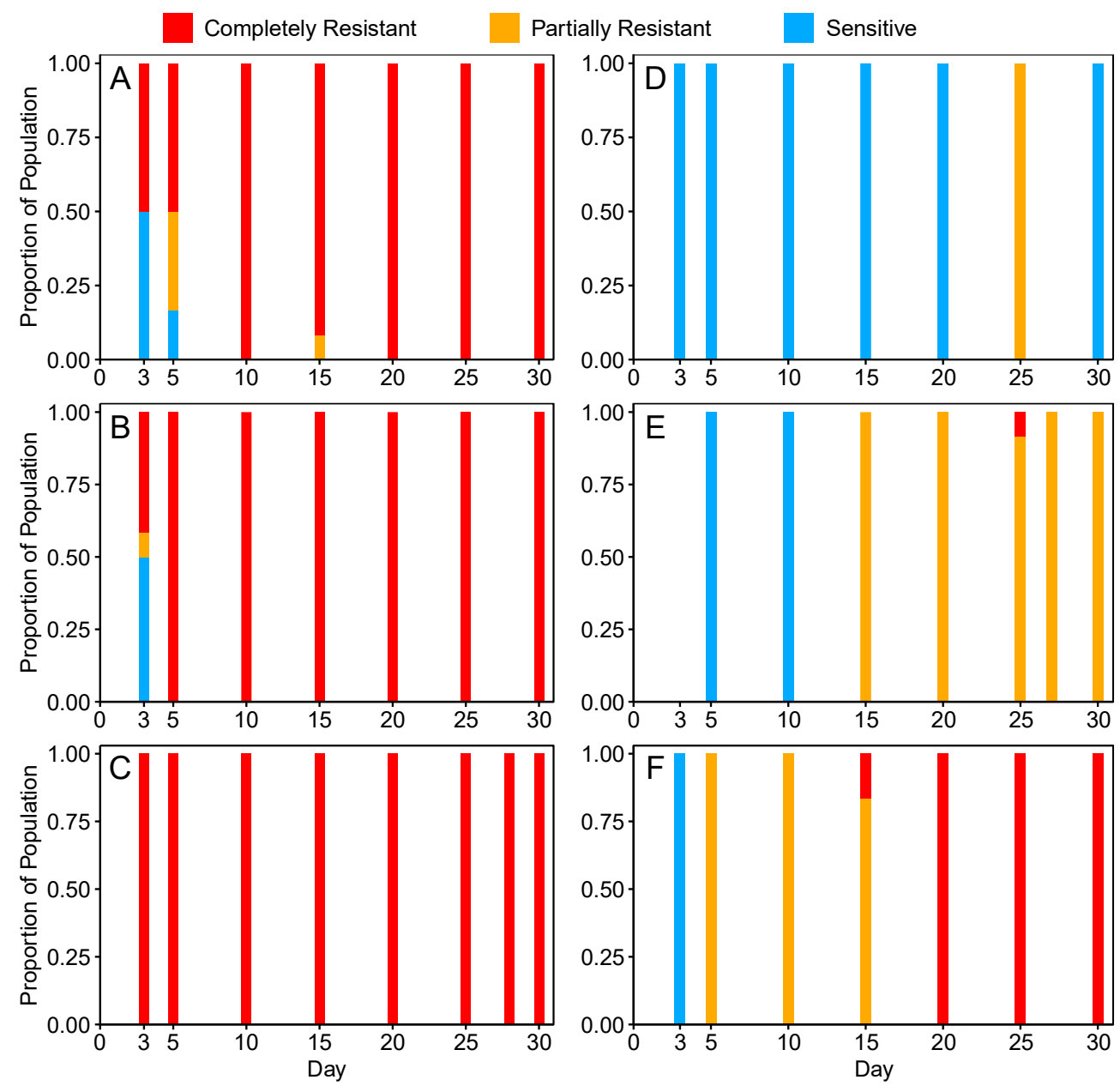

Figure 2. Proportion of sensitive (blue), partial (yellow) and complete (red) resistance of bacterial populations at various days of the coevolution experiment to ancestral phages from respective treatments $(n=12$ isolates per timepoint). $\lambda$ unt Populations 1-3 correspond to Panels A-C and $\lambda$ trn Populations 1-3 correspond to Panels D-F, respectively.

Mutation Rates of Resistance. In order to determine whether there are fewer accessible mutations for $\lambda$ trn resistance than for $\lambda$ unt resistance, we conducted Luria-Delbruck fluctuation tests. This protocol is used to estimate the rate of mutations that generate a particular phenotype, like resistance, by applying a selective screen and enumerating cells with the desired phenotype $(17,32)$. Because traditional fluctuation tests on agar plates could not detect some forms of resistance that evolved in our experiments (see Emergence of Resistance), we used a fluctuation test design suitable for liquid culture. Using the $\mathrm{P}_{0}$ method (32), we estimated a mutation rate for $\lambda$ unt resistance of $6.3 \times 10^{-6}$ per cell. With $\lambda$ trn, we found that the mutation rate for resistance was $2.2 \times 10^{-8}$ per cell, $\sim 100 \times$ lower than resistance to $\lambda$ unt. Notably, resistance to $\lambda$ unt appeared to confer complete resistance, whereas $\lambda$ trn resistance was only partial. Thus, mutations that confer $\lambda$ trn resistance are $\sim 100 \times$ less common than mutations for $\lambda$ unt resistance, and they only confer partial resistance.

Our inability to detect mutants with complete $\lambda$ trn resistance in fluctuation tests led us to focus on the second hypothesis, that complete resistance might require multiple mutations. To test this, 
we repeated fluctuation tests on 4 genetically distinct, partially resistant mutants isolated from the initial fluctuation test. We found that, across 10 replicate cultures for each isolate (40 total), only one replicate evolved complete resistance (with an additional mutation). Thus, under the artificial selection in fluctuation tests, multiple mutations were required to achieve complete $\lambda$ trn resistance. This led us to wonder whether complete resistance to $\lambda$ trn required multiple mutations in the coevolution experiment as well.

Genetics of Resistance. To identify the resistance mutations that evolved in the coevolution experiment, we sequenced whole genomes of representative isolates at the earliest time-point that resistance was detected, as well as of isolates at later time-points with distinct changes in resistance status (i.e. greater resistance or sensitivity; Fig. S1). We identified mutations using the computational pipeline breseq (33) and then classified putative resistance mutations as those that occurred in genes 1 ) known to be involved in $\lambda$ infection $(26,34)$ or 2 ) that evolved in parallel in multiple populations, fluctuation tests, or previous coevolution experiments $(26,35)$. In total, we found 2 unique putative resistance mutations across 4 bacterial isolates from flasks with $\lambda$ unt and 8 unique putative resistance mutations across 8 isolates from flasks with $\lambda$ trn (Fig. S3).

From flasks with $\lambda$ unt, all completely resistant day-3 isolates had 25 bp frameshift duplications in malT, a positive regulator of the receptor LamB, and no other putative resistance mutations (Fig. S3). Notably, isolates with partial $\lambda$ unt resistance only had point mutations in malT (e.g. isolate P1-T5-5-P, Fig. S1C, Fig. S3). Thus, whereas frame-shift mutations in malT conferred complete resistance to $\lambda$ unt, point mutations in malT were less protective. malT appeared to be the only locus to evolve resistance mutations in the $\lambda$ unt treatment.

In contrast, putative resistance mutations identified in the $\lambda$ trn treatment occurred at numerous loci. Many of the genes were not previously known to affect $\lambda$ infection; however, they were flagged because they evolved in parallel. For example, we found a 21,535 bp 27-gene deletion in all isolates from Population 2, as well as in a fluctuation test isolate (JB43). Moreover, JB42, an independently evolved partially resistant fluctuation test isolate, had a single mutation in $\operatorname{lpcA}$, which occurs within this $21,535 \mathrm{bp}$ range. Thus, we believe the large deletion observed in Population 2 isolates confers partial resistance by removing $l p c A$. The function of the gene suggests a mechanism for partial resistance; $l p c A$ codes for a phosphoheptose isomerase and is involved in assembly of the lipopolysaccharide (LPS) core and outer membrane biogenesis. It is possible that the disruption of $l p c A$ alters the organization of outer membrane proteins like LamB and $\mathrm{OmpF}$, which may interfere with phage adsorption. This hypothesis is also supported by the detection of parallel evolution in waa genes which are also associated with LPS core biogenesis. In both isolates from Population 3, we found a 4,894 bp IS1-mediated deletion of genes waaC through waaT and a nearby locus, waaO, evolved mutations in a previous experiment $(36,37)$. Previously, mutations in waa have only been associated with phage resistance insofar as those phages use LPS as a receptor $(36,37)$, however here they also appear to interfere with phages that use outer membrane proteins like LamB and OmpF. The mechanisms by which other putative resistance mutations confer partial $\lambda$ trn resistance are less clear and are discussed in SI Appendix: $\lambda$ trn Resistance.

After identifying putative resistance mutations that occurred in each bacterial isolate, we sought to quantify how these mutations affected phage resistance. Because strains possessed various 
compositions of mutations, we were unable to analyze their effects in isolation. Therefore, we conducted linear regressions to determine whether there was a relationship between the number of putative resistance mutations and the level of resistance to $\lambda$ unt or $\lambda \operatorname{trn}$. To discriminate between the resistance of different isolates (especially partially resistant isolates) we needed a way to quantitatively score resistance. We tried simple approaches to score resistance by analyzing the OD trajectories of cultures (e.g. computing the difference in the area under growth curves for bacteria without and with phage), however these methods were unable to characterize differences that were easily identifiable with the naked eye. When these approaches did not work, we used principal component analysis (PCA) on the growth trajectory data, which was able to transform and score growth trajectories along a continuous scale for resistance. As a result, principal component 1 ( $\mathrm{PC} 1,92.25 \%$ of variance) was able to discriminate sensitive, partial, and complete resistance, as well as between different levels of partial resistance (Fig. S4).

Using PC1 as an indicator of resistance, we conducted linear regressions to test the relationship between the number of putative resistance mutations and phenotypic resistance to ancestral phages (Fig. 3). We find that the relationship is significant for both $\lambda$ unt $(p=0.002)$ and $\lambda$ trn $(\mathrm{p}=0.003)$. The significant relationship for $\lambda$ trn suggests that the putative resistance mutations we identified played a role in building bacterial defenses. This observation combined with the fluctuation test analysis provides evidence in support of the second hypothesis that multiple mutations are required to confer complete resistance to $\lambda$ trn. All strains with complete resistance to $\lambda$ trn possessed five putative resistance mutations (Fig. 3B), whereas isolates with complete resistance to $\lambda$ unt had only one mutation (Fig. 3A). 


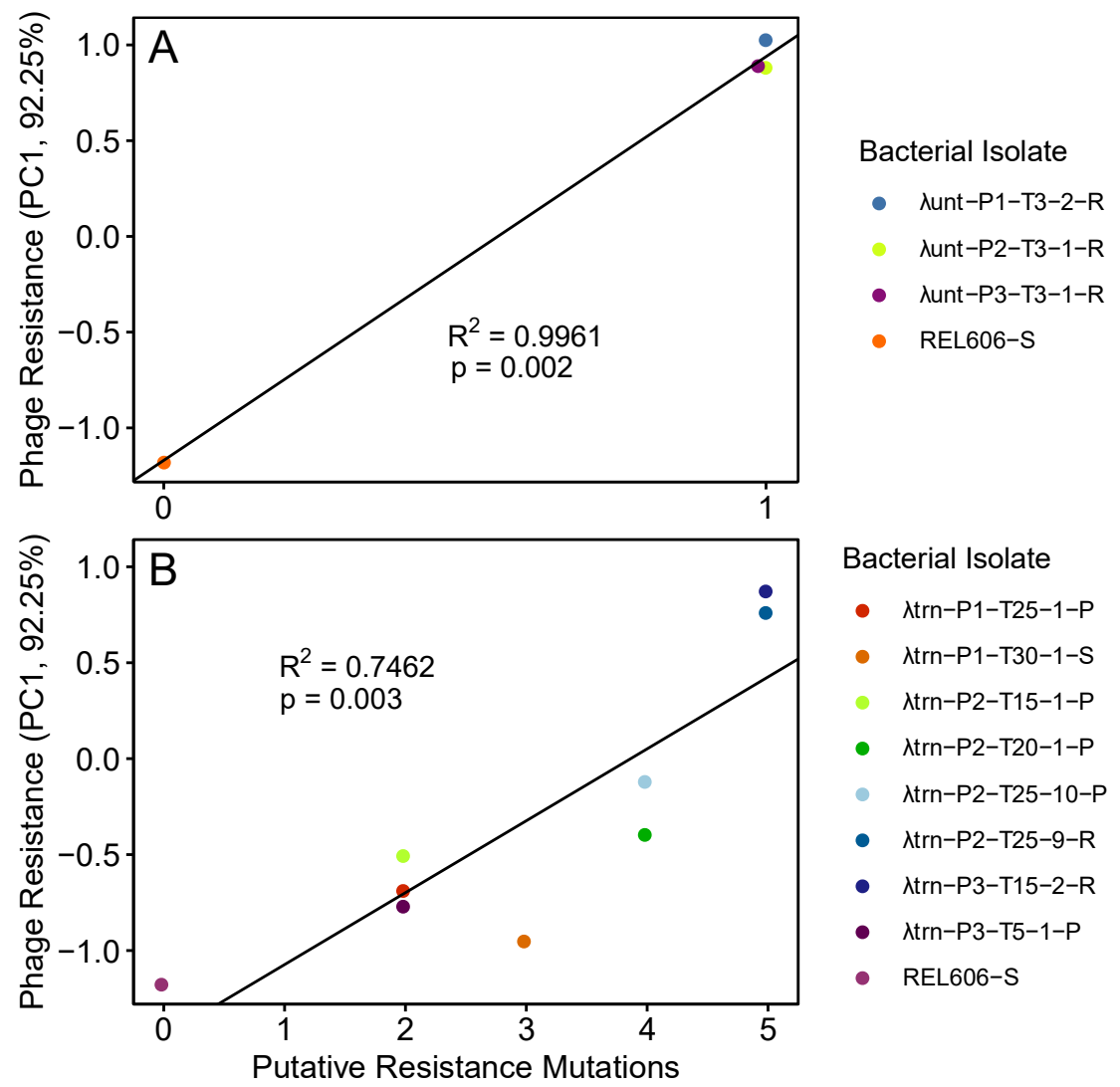

Figure 3. Relationship between the number of putative resistance mutations and the level of phage resistance (PC1 is indicative of resistance, see Fig. S4) from coevolutionary treatments with $\lambda$ unt (A) and $\lambda \operatorname{trn}(B)$. Linear regressions (PC1 Putative Resistance Mutations, black lines) are significant for both Panels A and B, however the number of mutations required for high levels of resistance is greater in B. Bacterial strains are labeled as [Treatment]-[Population]-[Day Isolated]-[Isolate \#]-[Resistance Status] where $\mathrm{S}=$ Sensitive, $\mathrm{R}=$ Complete Resistance, and $\mathrm{P}=$ Partial Resistance to phages from respective treatments.

Costs of Resistance. In order to determine whether resistance to $\lambda$ trn was costlier than to $\lambda$ unt, we conducted competition experiments where resistant bacteria with different genotypes were paired with a genetically marked sensitive strain (Fig. 4). $\lambda$ unt resistance carried only a small cost compared to the marked competitor $(\bar{w}=0.972, \mathrm{t}=-3.8617, \mathrm{p}=0.005$, one-sample $\mathrm{t}$-test $)$. This was expected because malT mutants cultured in similar laboratory conditions were not found to bear a cost in previous studies $(38,39)$. In contrast, resistance to $\lambda$ trn was more costly than $\lambda$ unt resistance $(\bar{w}=0.596, \mathrm{t}=-8.19, \mathrm{p}<<0.001$, two-sample $\mathrm{t}$-test $)$. The fitness burden was similar regardless of whether the mutations conferred partial or complete resistance $(p=0.67$, twosample t-test). Thus, any level of resistance to $\lambda$ trn has a cost, which helps explain the lag in the development of resistance to this phage (Fig. 1A). 


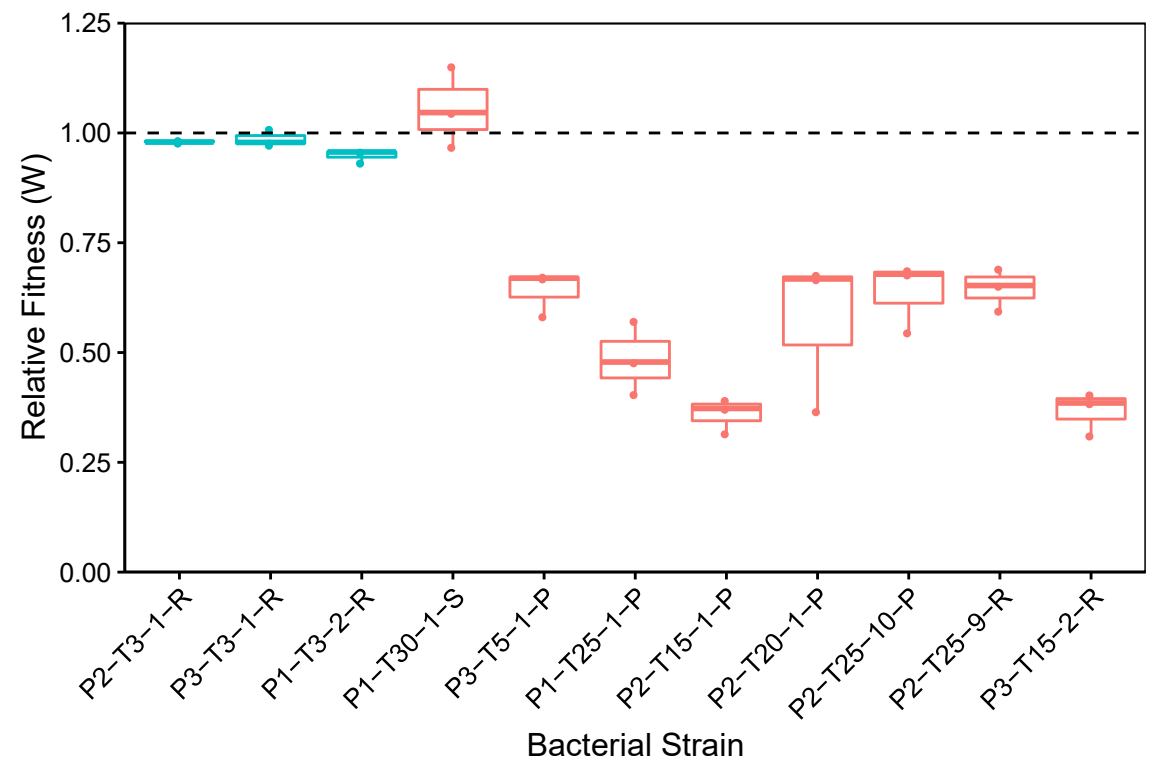

Figure 4. Fitness of coevolved bacterial isolates relative to their common ancestor, REL606. Isolates that coevolved with $\lambda$ unt are in teal and $\lambda \operatorname{trn}$ in red. Bacteria are labeled as in Fig. 3 except with treatment omitted. Mean relative fitness is significantly different between treatments with $\lambda$ unt $(\bar{w}=0.972)$ and $\lambda \operatorname{trn}(\bar{w}=0.596$, independent twosample t-test, $\mathrm{t}=-8.19, \mathrm{p}<<0.001)$. However, the relative fitness of isolates with partial and complete resistance to $\lambda \operatorname{trn}$ does not differ $(\mathrm{p}=0.67)$. Strains are ordered firstly by treatment $(\lambda u n t, \lambda \operatorname{trn})$ and then by increasing resistance according to PC1 (see Fig. 3).

Phage Adaptation and Suppression of Resistance. Thus far, we have focused on the bacterial response to $\lambda$ unt and $\lambda$ trn treatments. However, the ability of phages to evolve counter resistance to their hosts could have also contributed to differences in bacterial suppression and slowed the progression of resistance. To investigate whether phages evolved counter-defenses, we isolated a single phage from each population on the day we first detected that co-occurring bacteria had evolved resistance. We refer to co-occurring phage isolates as $\lambda$ unt+ and $\lambda \operatorname{trn}+$ to differentiate them from their ancestors. We then conducted plate reader experiments to determine whether cooccurring phages ( $\lambda$ unt + or $\lambda$ trn + ) had evolved enhanced ability to suppress the earliest resistant bacteria to emerge from each population compared to ancestral phages ( $\lambda$ unt or $\lambda$ trn). We found that $\lambda$ unt+ phages were no better than $\lambda$ unt at suppressing resistant hosts (Fig. S5).

Contrastingly, all $\lambda$ trn + phages were better able to suppress co-occurring, resistant hosts than their ancestor, $\lambda \operatorname{trn}$ (Fig. 5). For Population 1, we compared the suppression of host P1-T25-1-P by $\lambda \operatorname{trn}$ and $\lambda \operatorname{trn}-\mathrm{P} 1-\mathrm{T} 25$. We found greater suppression by $\lambda \operatorname{trn}-\mathrm{P} 1-\mathrm{T} 25$, apparent visually (Fig. 5A), as well as quantitatively by comparing PC1 between replicate cultures (Fig. 5B, p=0.004, two-sample $t$-test). The same was true for $\lambda$ trn + phages from Population $2(\mathrm{p}=0.017$, two-sample t-test, Fig. 5D, 5E) and Population 3 (p=0.034, two-sample t-test, Fig. 5G, 5H). For each population, comparisons were drawn from cultures where phage inoculum densities were not significantly different (SI Appendix: Phage Adaptation and Suppression of Resistance).

Support for these results also comes from efficiency of plating (EOP) assays. EOP, the ratio of plaques that a phage lysate forms on different bacteria, is often used to indicate how well phage are adapted to different hosts. All $\lambda$ trn+ phages had higher EOP on resistant hosts than $\lambda$ trn (Fig. 5C, 5F, 5I, $\mathrm{p}=0.022, \mathrm{p}=0.045, \mathrm{p}=0.037$, respectively, two-sample t-tests). Although EOP alone is 
not indicative of improved suppression, these results support our conclusion that $\lambda \operatorname{trn}+$ phages adapted to better suppress resistant hosts, whereas adaptation of $\lambda$ unt + phages did not improve suppression. Two possibilities explain these results; $\lambda$ trn may adapt to hosts more quickly and/or the kinds of resistance that evolved against $\lambda$ trn may have been easier for the phage to counter. We did not investigate this further and do not know which contributed to our results.
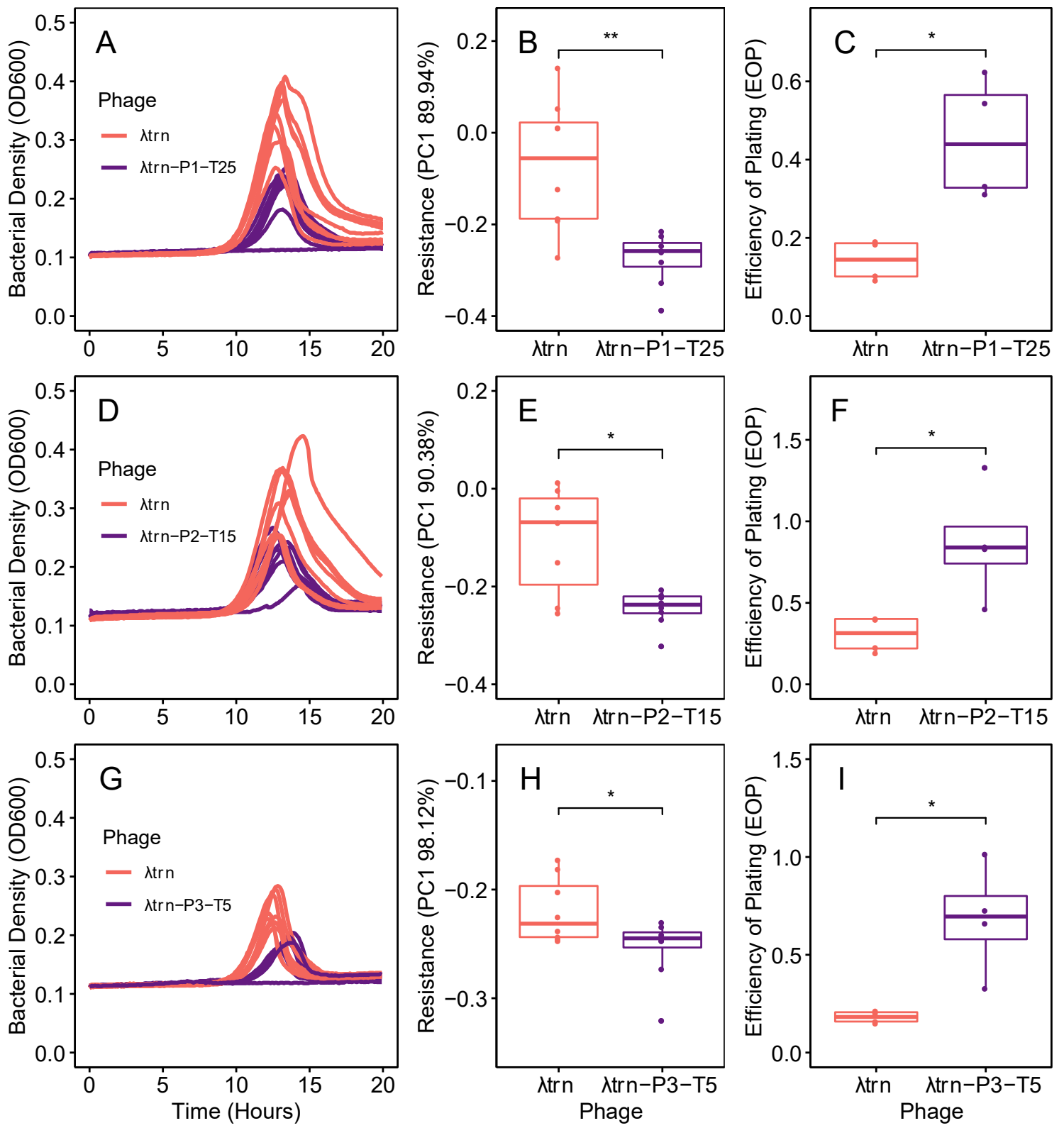

Figure 5. Coevolved $\lambda$ trn + isolates show adaptation and improved suppression of phage resistant bacteria. In all panels, comparisons are between ancestral phage $\lambda \operatorname{trn}(\mathrm{red})$ and a contemporary $\lambda$ trn + phage (purple) isolated from the same population and time-point as the resistant bacterial isolate. Panels A-C pertain to host P1-T25-1-P. Panels D-F pertain to host P2-T15-1-P. Panels G-I pertain to host P3-T5-1-P. Bacterial isolates are labeled as in Fig. 4. All hosts represent the first day at which $\lambda$ trn resistance was detected. Panels A, D, G: Growth trajectories of replicate wells $(n=4)$ inoculated with $\sim 1000$ cells of the resistant host and either $\lambda \operatorname{trn}$ or $\lambda$ trn + phage. Panels B, E, H:

Resistance of bacterial isolates to $\lambda \operatorname{trn}$ and respective $\lambda \operatorname{trn}+$ phage (indicated by PC1, see Fig. S4). In all populations, bacterial isolates are less resistant to $\lambda \operatorname{trn}+$ phages than to $\lambda \operatorname{trn}$ (Two-sample t-tests; $B, p=0.004 ; E, p=0.017 ; H$, 
$\mathrm{p}=0.034)$. Panels C, F, I: Efficiency of plating (EOP) of $\lambda$ trn and $\lambda$ trn+ phages on respective, resistant hosts compared to on REL606. In all populations, EOP is higher for $\lambda \operatorname{trn}+$ phages than $\lambda \operatorname{trn}(C, p=0.022 ; F, p=0.045 ; I$, $\mathrm{p}=0.037)$.

$\lambda$ trn Adaptation via Recombination. At this point in the study, we explored how phage training enabled $\lambda$ trn to gain its suppressive abilities (Table S1). Previously, it was reported that five mutations in $\lambda$ 's ( $\lambda$ unt's) host recognition gene $J$ gave it the ability to use the new receptor, OmpF (26). In the same gene, there is an enigmatic $\sim 1,300 \mathrm{bp}$ region that experienced 49 mutations during phage training. These mutations appear to be the result of recombination between $\lambda$ 's $J$ gene (1545-2796) and REL606's ECB_00512 gene (882-2217). ECB_00512 shares homology with $J$ and $E C B \_00512$ is likely a genetic relict from past phage infection. The appearance of recombination in a gene under selection led us to question whether the recombination was adaptive. Using a previously reported phylogeny of the experiment in which the recombination evolved (35), we were able to deduce when the recombination occurred and engineer $J$ alleles before and after the recombination. Next, we inserted the alleles and a genetic marker into $\lambda$ unt so that we could run competition experiments between pre- and postrecombination phages. We found that the recombination increased $\lambda$ unt's ability to compete for wildtype (REL606), malT-, and malT-OmpF- host cells (Fig. 6; paired t-test of $\lambda$ growth rates, pvalues for wildtype, malT-, and malT-OmpF- were $0.002,0.006$, and 0.004 , respectively). The recombination had the largest effect in competitions for malT-ompF-cells, which were the most difficult to infect and in which phage fitness doubled (ANOVA, $\mathrm{p}=0.005$; Tukey's Test $\mathrm{p}<0.05$ ).

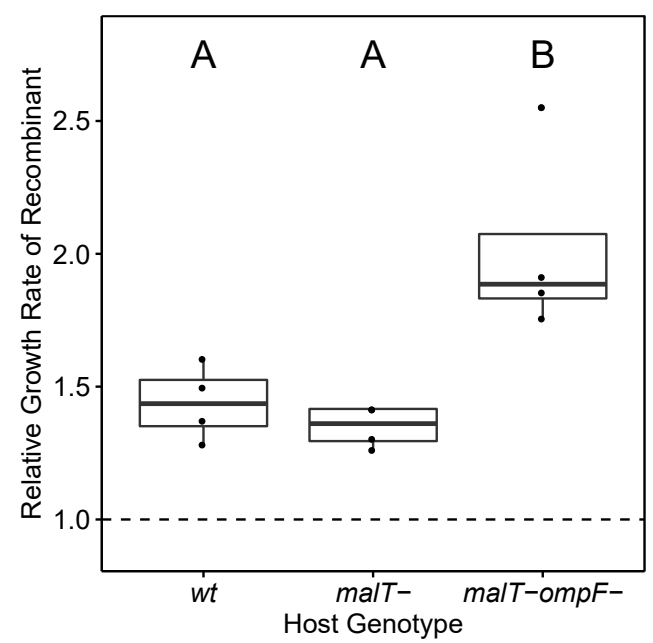

Figure 6. Increase in exponential growth rate of recombinant $\lambda$, relative to pre-recombination $\lambda$, when competing for three different $E$. coli host genotypes. Letters indicate groups that are statistically similar. The dashed line indicates where growth rate of the recombinant is equal to pre-recombination $\lambda$.

We find it remarkable that, through recombination, the relict DNA of a past infection provided information to improve infectivity of our contemporary phage. We expected that phage training worked entirely through the steady processes of coevolution, in which the phage makes incremental, reciprocal advances on its evolving host. However, recombination may accelerate training by transferring information from past phages that were well adapted to their host to new, naïve therapeutic phages. This may be an important mechanism for phage training success because therapeutic phages are typically isolated independently from the target bacterium $(9,11,13)$ and may not be well adapted to these hosts. Many, if not most bacteria possess genes 
that originated from phage genomes $(22,40)$ and phage recombination is frequently found in nature (41-43). Moreover, a previous study found that recombination with host encoded phages resulted in a host-range expansion (41), suggesting that recombination could be a dependable mechanism that enhances the effectiveness of phage training beyond the coevolutionary arms race. While the use of phage with the potential to recombine has generally been avoided due to the risk of transducing bacterial virulence or antibiotic resistance genes (43), here we show a potential benefit of phage-host recombination in phage training. If recombination is a concern during phage therapy, the recombination machinery could be left intact during training and then knocked out before treatment.

\section{Discussion}

In phage therapy, the evolution of phage resistance is widespread and often renders phage therapeutics ineffective. Here, we evaluated the prospect of coevolutionary phage training as an approach to improve therapeutic efficacy by comparing untrained ( $\lambda$ unt) and trained $(\lambda \operatorname{trn})$ phages cocultured with a naïve target bacterium. We found that treatment with $\lambda \operatorname{trn}$ led to improved and prolonged suppression of host populations and that the evolution of $\lambda$ trn resistance was delayed as compared to treatment with $\lambda$ unt. This prolonged suppression and delayed resistance was caused by several factors that all support coevolutionary phage training as an approach that could improve outcomes in phage therapy.

We found that it was more difficult for bacteria to evolve resistance to trained phage. Firstly, whereas individual mutations conferred complete resistance to $\lambda$ unt, mutations for $\lambda$ trn resistance were $\sim 100 \times$ less common and only conferred partial resistance. Secondly, complete $\lambda$ trn resistance required multiple mutations and both completely resistant bacteria isolated from the coevolution experiment had five putative resistance mutations. We believe the reduced ability of bacteria to evolve resistance to $\lambda$ trn than to $\lambda$ unt was the greatest contributor to the stronger suppression and delayed resistance observed in the coevolution experiment. In part, these differences likely originate from the fact that $\lambda$ trn can use two receptors (LamB and OmpF), whereas $\lambda$ unt can only use LamB. Phage resistance often evolves through augmentations of the receptors, and so deploying a phage with a backup receptor would be similar to using a drug cocktail where multiple mutations are required for bacteria to survive the treatment. It is unclear whether evolving to use a new receptor is a typical outcome of phage training; nonetheless, the use of dual receptor phages should be considered in developing future strategies for phage discovery (44), engineering $(45,46)$, and directed evolution.

Thirdly, we found that $\lambda$ trn resistance mutations carried large growth costs, regardless of whether they conferred partial or complete resistance, whereas mutations for $\lambda$ unt resistance carried little cost. In conjunction with the fact that, initially, resistance to $\lambda$ trn is only partial, these large growth costs appear to have slowed the rate at which bacterial populations reached carrying capacity. Together, these factors may improve the ability of trained phage therapeutics, in combination with the host immune system, to resolve infections (47).

And fourthly, we found that previously trained phages were better able to counter the evolution of resistance during the experiment. In all populations, coevolved $\lambda$ trn + phages, isolated from the same time-point at which resistance was detected, were better able to suppress and had higher 
EOP on partially resistant hosts than their ancestor, $\lambda$ trn. This was not true for coevolved $\lambda$ unt + phages, which lost the ability to suppress resistant hosts. Although the role of phage coevolution in the efficacy of in vivo therapy has yet to be demonstrated, the ability of trained phages to coevolve with and counter host defenses may improve treatment outcomes.

Despite the close relatedness of the phages that we used, the effects of $\lambda$ trn on the ecological and evolutionary dynamics of the microbial communities was vastly different from $\lambda$ unt. Flasks with $\lambda$ unt shifted from top-down (predator-controlled) to bottom-up (resource limited) systems where bacterial populations were limited by the availability of glucose in less than one day. With $\lambda$ trn, flasks persisted as top-down systems in which phages suppressed bacterial populations below carrying capacity for several weeks. Moreover, the genetics underlying E. coli's resistance evolution was very different. With untrained phage, bacteria acquired single, large-effect mutations that conferred complete resistance to $\lambda$ unt. However, with a trained phage, bacteria were constrained to evolve resistance through multiple mutations that each only yields partial resistance. These observations are fascinating from a basic biology perspective because they show how slight alterations in genomes and the evolutionary history of parasites can change their host's ecology and evolution. From an applied standpoint, these observations provide proof of principle that directed evolution can vastly improve the effectiveness of phage therapeutics. Phage malleability is often lauded as a potential strength of phage therapy, and this is strongly supported by our findings.

Altogether, these results contribute to a small but growing body of work $(23,48,49)$ in support of coevolutionary phage training as a means to improve the efficacy of phage therapeutics. Furthermore, they reveal the mechanisms by which this improved efficacy can be achieved: bacteria appear less able to resist trained phage and trained phage are more able to adapt to counter their hosts. We encourage efforts to investigate phage training on clinical strains of bacteria and to study the efficacy of trained phages in the treatment of animal model infections. 


\begin{abstract}
Methods
Strains. To study the effects of phage training on bacteria-phage coevolutionary dynamics we used Escherichia coli B strain REL606, as well as untrained ( $\lambda$ unt) and trained ( $\lambda$ trn) strains of phage $\lambda$. For $\lambda$ unt, we used phage $\lambda$ cI26, a strictly lytic form of $\lambda$ that has been characterized and compared with the $\lambda$ reference genome by Meyer et al. (26). For $\lambda$ trn, we used phage $\lambda 28-11$, a descendant of $\lambda$ cI26 that was isolated from a plaque after coevolving with REL606 for 28 days $(26,35)$. Notably, $\lambda$ trn can infect through the native $\lambda$ receptor, LamB, as well as a novel receptor, OmpF. We consider $\lambda$ trn trained because it has previously coevolved with host REL606. Table S1 shows genetic comparisons between $\lambda$ trn and $\lambda$ unt.
\end{abstract}

Bacterial Suppression experiment. To determine whether trained ( $\lambda$ trn) phages would better suppress REL606 than untrained ( $\lambda$ unt) phages, we inoculated six replicate $50 \mathrm{~mL}$ flasks with $10 \mathrm{~mL}$ modified $\mathrm{M} 9$ glucose media (M9-G, recipe in (26)) and $\sim 10^{4}$ cells. Of these flasks, 3 were inoculated with $\sim 10^{8}$ particles of $\lambda$ unt and 3 with $\sim 10^{7}$ particles of $\lambda \operatorname{trn}$. Flasks were incubated at $37^{\circ} \mathrm{C}$, shaking at $120 \mathrm{rpm}$. After $24 \mathrm{~h}, 100 \mu \mathrm{L}$ of each community was transferred into new flasks with $10 \mathrm{~mL}$ of fresh media. Flasks were propagated for 30 days. Each day, aliquots were removed to estimate bacterial and phage densities, as well as to preserve communities for later analyses. For bacteria, aliquots were diluted in M9-G with $0.01 \mathrm{M}$ sodium citrate and plated on Luria-Bertani (LB) agar. For phages, $1 \mathrm{ml}$ aliquots were treated with chloroform $(5 \% \mathrm{v} / \mathrm{v})$ and centrifuged $(1 \mathrm{~min}$ at $15,000 \mathrm{x} g)$ to extract lysates. Then, lysates were diluted in M9-G and $2 \mu \mathrm{L}$ aliquots were spotted on infused soft agar (LB agar except with $0.8 \%$ w/w agar and inoculated with $\sim 10^{8}$ cells REL606 (26)). Lastly, starting on day 3, aliquots were preserved by freezing at $-80^{\circ} \mathrm{C}$ in $15 \% \mathrm{v} / \mathrm{v}$ glycerol.

Bacterial and Phage Isolation. To isolate bacteria, scrapes $(\sim 2 \mu \mathrm{L})$ of preserved, frozen communities were streaked on LB agar plates and incubated overnight at $37^{\circ} \mathrm{C}$. Then, colonies were isolated and streaked twice more to obtain clonal strains cleaned of phage. Strains were grown overnight at $37^{\circ} \mathrm{C}$ in $\mathrm{M} 9-\mathrm{G}$ and preserved by freezing. To isolate phages, scrapes of ice were suspended in tubes with $1 \mathrm{~mL}$ of M9-G. Then $10 \mu \mathrm{L}$ was inoculated in molten $\left(\sim 55^{\circ} \mathrm{C}\right)$ infused soft agar, poured over LB agar plates, and incubated overnight at $37^{\circ} \mathrm{C}$. From these plates, plaques were isolated and plated in infused soft agar two more times. Then, plaques were picked into tubes with $4 \mathrm{~mL}$ of M9-G and $\sim 10^{8}$ cells REL606 and incubated overnight at $37^{\circ} \mathrm{C}$. Lastly, lysates were extracted and preserved as described above.

Emergence of Resistance. To determine when resistance evolved to $\lambda$ unt and $\lambda$ trn, we used 12 bacterial isolates from each community across various days of the coevolution experiment. We conducted resistance assays in liquid using 96-well plates and a plate reader (Tecan Sunrise). For each isolate, overnight cultures in LB were diluted and $\sim 10^{3}$ cells inoculated into wells containing either $200 \mu \mathrm{L}$ of LBM9 or $150 \mu \mathrm{L}$ of LBM9 with $50 \mu \mathrm{L}$ of $0.22 \mu \mathrm{m}$ filtered lysate prepared in LBM9 $\left(\sim 10^{8}\right.$ phage particles, recipe in (27)). Optical density (OD) at wavelength $600 \mathrm{~nm}$ was read from plates over $20 \mathrm{~h}$ of incubation, shaking at $37^{\circ} \mathrm{C}$. We used LBM9 instead of M9-G for plate reader assays because experiments in M9-G were unable to distinguish between sensitivity and resistance against $\lambda$ unt. In M9-G, the native $\lambda$ receptor, LamB, is downregulated and $\lambda$ unt does not adsorb to nor kill cells efficiently $(50,51)$. In contrast, LBM9 allowed us to distinguish sensitive, partial, and complete resistance.

Estimating Resistance Mutation Rate. To estimate REL606's mutation rate for resistance $\left(\mu_{\mathrm{r}}\right)$ against $\lambda$ unt and $\lambda$ trn, we conducted Luria-Delbruck fluctuation tests in liquid culture. REL606 was grown in LB overnight, diluted, inoculated into independent isogenic cultures $\left(\sim 10^{3}\right.$ cells per well) in a 96-well plate, and grown again overnight. Cultures were diluted and $10 \mu \mathrm{L}$ inoculated into a new plate with $150 \mu \mathrm{L}$ of LBM9 and $50 \mu \mathrm{L}$ of filtered LMB9 lysate $\left(\sim 10^{8}\right.$ phage particles) in each well. To estimate $\mu_{\mathrm{r}}$ to $\lambda$ unt, fluctuation tests were conducted with $10^{4}$ - and $10^{5}$ cell inoculums. To estimate $\mu_{\mathrm{r}}$ to $\lambda$ trn, we used $10^{7}$-cell inoculums. Plates were read for $20 \mathrm{~h}$ while shaking at $37^{\circ} \mathrm{C}$. Wells with no observable growth were deemed sensitive. The $\mathrm{P}_{0}$ method $\left(\mathrm{P}_{0}=e^{\mu \mathrm{r}^{*}(\mathrm{~N}-\mathrm{No})}\right)$ was used to calculate $\mu_{\mathrm{r}}$, where $\mathrm{P}_{0}$ is the proportion of wells that were sensitive, $\mathrm{N}$ is the number of cells inoculated in each well, and No is the number of cells used to start cultures $\left(\sim 10^{3}\right.$ cells $)$. To screen for false positives, bacteria were isolated from wells showing resistance and tested in 96-well plates as described above.

Bacterial and phage genomics. To extract genomes, bacteria were streaked from frozen stocks onto LB agar and incubated overnight. Colonies were picked into respective growth media (M9-G for coevolution, LB for fluctuation test), incubated overnight, and genomes were extracted using an Invitrogen PureLink Genomic DNA Mini Kit. For phages, lysates were prepared by incubating $\sim 2 \mu \mathrm{L}$ from frozen stocks with $\sim 10^{8}$ cells of REL606 overnight in M9- 
$\mathrm{G}$ and then extracted using $0.22 \mu \mathrm{m}$ filters. To extract genomes, $500 \mu \mathrm{L}$ of lysate was transferred to $1.5 \mathrm{~mL}$ microcentrifuge tubes with $50 \mu \mathrm{L}$ of DNase buffer (10x), $10 \mathrm{U}$ of DNase I, and $1 \mu \mathrm{L}$ of $100 \mathrm{mg} / \mathrm{mL}$ RNaseA and incubated for $30 \mathrm{~min}$ at room temperature. Then, $20 \mu \mathrm{L}$ of $0.5 \mathrm{M}$ EDTA, $2.5 \mathrm{U}$ of proteinase $\mathrm{K}$, and $25 \mu \mathrm{L}$ of $10 \%$ SDS were added and tubes incubated at $55^{\circ} \mathrm{C}$ for $1 \mathrm{~h}$. Lastly, phenol chloroform extractions were conducted to purify the phage DNA [52]. The resulting supernatant and an equal volume of $100 \% \mathrm{EtOH}$ were added to spin columns from a Purelink Genomic DNA Mini Kit and then washed and eluted according to the manufacturer's instructions. Finally, extracted bacterial and phage genomes were sent to the Microbial Genome Sequencing Center (MiGS) where they were indexed and sequenced on an Illumina NextSeq 550. Sequences were analyzed using breseq (version 0.35.0), a computational pipeline for analyzing short-read re-sequencing data [33].

Genetics of Resistance. To investigate the relationship between the number of resistance mutations and the level of resistance in bacterial isolates, we first identified resistance mutations as those occurring in genes that are involved in $\lambda$ infection or that evolved in multiple populations, fluctuation tests, or previous coevolution experiments. Then, we created a scale indicative of resistance by taking the difference in OD trajectories of bacterial cultures growing without and with phage and conducting principal component analysis (PCA) on these OD difference vectors in R (version 3.6.1, (53)). PCA revealed that PC1 explained $92.25 \%$ of the variance and discriminated between sensitive, partial, and complete resistance along a continuous scale. Using PC1 values as resistance scores, we used R to implement linear models (PC1 number of resistance mutations) testing the relationship between mutations and resistance against each phage.

Costs of Phage Resistance. Competitions were conducted between coevolved bacterial isolates and an ara+ marked REL606 ancestor. To obtain the marked ancestor, $\sim 10^{8}$ cells of REL606 (ara-) were plated on minimal-arabinose agar plates (5.34 g potassium phosphate dibasic anhydrous, $2 \mathrm{~g}$ potassium phosphate monobasic anhydrous, $1 \mathrm{~g}$ ammonium sulfate, $0.57 \mathrm{~g}$ sodium citrate dihydrate, $16 \mathrm{~g}$ agar, $4 \mathrm{~g}$ arabinose per liter of water and supplemented to a final concentration of $1 \mathrm{mM}$ magnesium sulfate, $0.0002 \% \mathrm{w} / \mathrm{v}$ thiamine, and $0.0002 \% \mathrm{w} / \mathrm{v}$ biotin). After 2 days of incubation at $37^{\circ} \mathrm{C}$, colonies were picked and streaked twice on tetrazolium-arabinose indicator agar (Tet-ara) plates (10 g tryptone, $1 \mathrm{~g}$ yeast extract, $5 \mathrm{~g}$ sodium chloride, $16 \mathrm{~g}$ agar, $10 \mathrm{~g}$ arabinose per liter of water and supplemented to a final concentration of $0.005 \%$ tetrazolium indicator dye TTC) to confirm marker presence and obtain isogenic stocks. To initiate competitions, colonies on Tet-ara plates were picked and incubated for $24 \mathrm{~h}$ as in the coevolution experiment. The next day, coevolved strains and the marked competitor were inoculated 1:9 or 1:99 to a final volume of $100 \mu \mathrm{L}$ in fresh media. Upon inoculation, flasks were mixed, and an aliquot was diluted and plated on Tet-ara plates to enumerate initial densities $\left(\mathrm{T}_{0}\right)$ of each strain. After competing at $37^{\circ} \mathrm{C}$ for $24 \mathrm{~h}$, aliquots were again diluted and plated on Tet-ara plates for final densities $\left(\mathrm{T}_{\mathrm{F}}\right)$. Finally, relative fitness $(\mathrm{W})$ was calculated for each strain where $\mathrm{W}=\mathrm{M}_{\mathrm{A}} / \mathrm{M}_{\mathrm{B}}$ and where $\mathrm{M}_{\mathrm{A}}=\ln \left[\mathrm{T}_{\mathrm{F}} / \mathrm{T}_{0}\right]$ of the coevolved strain and $\mathrm{M}_{\mathrm{B}}=\ln \left[\mathrm{T}_{\mathrm{F}} / \mathrm{T}_{0}\right]$ of the marked ancestor.

Phage Adaptation and Suppression of Resistance. First, coevolved phages were isolated from frozen communities as described above. Then, plate reader experiments were conducted as described in Emergence of Resistance except with the following changes: phage lysates were prepared on REL606 but 96-well plates were inoculated with representative resistant bacteria. Ancestral ( $\lambda$ unt, $\lambda \operatorname{trn})$ and coevolved phages $(\lambda u n t+, \lambda \operatorname{trn}+)$ were inoculated at various dilutions and were also enumerated by spotting $2 \mu \mathrm{L}$ of the lysate dilution series on soft agar plates infused with REL606 in quadruplicate. Then, PCA was conducted on OD growth trajectories of bacteriaphage cocultures where phage inoculum densities were not significantly different between treatments. We also spotted $2 \mu \mathrm{L}$ of the dilution series on resistant isolates to calculate efficiency of plating (EOP) on resistant hosts where $\mathrm{EOP}=$ [titer on resistant host] / [titer on REL606].

Phage Adaptation via Recombination. We constructed 4 phage strains from $\lambda$ unt with pre- and post-recombination $J$ alleles, both with and without a lacZ genetic marker, in order to isolate the effect of the $J$ mutations. Using a previously constructed phylogeny of the experiment in which the recombination evolved (35), we determined that the recombination occurred within a $J$ allele that had already evolved 7 mutations (C2999T, A3034G, T3230, C3310, G3319A, T3321A, A3364T). Therefore, we constructed alleles with these 7 mutations with and without the recombination. Fortunately, the 7 mutations do not overlap with the recombination, so we were able to PCR amplify DNA fragments with either the 7 mutations or the entire gene using the primers in Table S2. These fragments were ligated into a plasmid using an Invitrogen TA Cloning ${ }^{\mathrm{TM}}$ Kit and the plasmids were used to transform E. coli DH5 $\alpha$. $\lambda$ unt was modified by infecting these cells and then screening for particles that could use the non-native OmpF receptor as a result of incorporating the $J$ alleles from the plasmid. We constructed two independent versions of each 
phage genotype and the incorporation of the desired alleles was sequence verified. Next, we added a lac $Z$ genetic marker which causes the phage to produce blue plaques $(54,55)$. Head-to-head competitions were performed in conditions identical to the original evolution experiment (26). Competitions for 3 different hosts were evaluated; wildtype (REL606), malT- (REL606 with a 25 bp duplication in malT), and malT-ompF- (malT- with a single base substitution resulting in an amino acid change (N52K) in ompF (full genome in (26))). Four replicates were performed for each competition. Half of the competitions were conducted with pre-recombination genotypes marked with lac $Z$ and the other half with the post-recombination genotype marked. Each flask was initiated with $\sim 10^{6}$ particles of each $\lambda$ genotype and $\sim 10^{8}$ cells. Initial and final phage densities were enumerated by counting plates on $\mathrm{X}$-gal agar indicator plates (recipe in (54)).

Acknowledgements. We thank members Sarah Medina and Elijah Horwitz of the J.R.M. laboratory for productive discussions and feedback on this study. This work was supported by the United States - Israel Binational Science Foundation: 2017056 and the National Institutes of Health (NIH) Grant R01: GM088344. 


\section{References}

1. Interagency Coordination Group on Antimicrobial Resistance. No time to wait: securing the future from drug-resistant infections. Report to the secretary-general of the United Nations. Geneva: World Health Organization; 2019. https://www.who.int/antimicrobial-resistance/interagency-coordination-group/finalreport/en/. Accessed 11 March 2020.

2. Levy, S.B. and Marshall, B., 2004. Antibacterial resistance worldwide: causes, challenges, and responses. Nature medicine, 10(12), pp.S122-S129.

3. Davies, J. and Davies, D., 2010. Origins and evolution of antibiotic resistance. Microbiol. Mol. Biol. Rev., 74(3), pp.417-433. 6.

4. Zhang, X.X., Zhang, T. and Fang, H.H., 2009. Antibiotic resistance genes in water environment. Applied microbiology and biotechnology, 82(3), pp.397-414.

5. Cooper, M.A. and Shlaes, D., 2011. Fix the antibiotics pipeline. Nature, 472(7341), pp.32-32. 12.

6. Tommasi, R., Brown, D.G., Walkup, G.K., Manchester, J.I. and Miller, A.A., 2015. ESKAPEing the labyrinth of antibacterial discovery. Nature reviews Drug discovery, 14(8), pp.529-542.

7. Kortright, K.E., Chan, B.K., Koff, J.L. and Turner, P.E., 2019. Phage therapy: a renewed approach to combat antibiotic resistant bacteria. Cell host \& microbe, 25(2), pp.219-232.

8. Schooley, R.T. and Strathdee, S., 2020. Treat phage like living antibiotics. Nature Microbiology, 5(3), pp.391-392.

9. Schooley, R.T., Biswas, B., Gill, J.J., Hernandez-Morales, A., Lancaster, J., Lessor, L., Barr, J.J., Reed, S.L., Rohwer, F., Benler, S. and Segall, A.M., 2017. Development and use of personalized bacteriophagebased therapeutic cocktails to treat a patient with a disseminated resistant Acinetobacter baumannii infection. Antimicrobial agents and chemotherapy, 61(10), pp.e00954-17.

10. Dedrick, R.M., Guerrero-Bustamante, C.A., Garlena, R.A., Russell, D.A., Ford, K., Harris, K., Gilmour, K.C., Soothill, J., Jacobs-Sera, D., Schooley, R.T. and Hatfull, G.F., 2019. Engineered bacteriophages for treatment of a patient with a disseminated drug-resistant Mycobacterium abscessus. Nature medicine, 25(5), pp.730-733.

11. Aslam, S., Courtwright, A.M., Koval, C., Lehman, S.M., Morales, S., Furr, C.L.L., Rosas, F., Brownstein, M.J., Fackler, J.R., Sisson, B.M. and Biswas, B., 2019. Early clinical experience of bacteriophage therapy in 3 lung transplant recipients. American Journal of Transplantation, 19(9), pp.2631-2639.

12. Torres-Barceló, C., 2018. Phage therapy faces evolutionary challenges. Viruses, 10(6), p.323. 19.

13. Chan, B.K., Turner, P.E., Kim, S., Mojibian, H.R., Elefteriades, J.A. and Narayan, D., 2018. Phage treatment of an aortic graft infected with Pseudomonas aeruginosa. Evolution, medicine, and public health, 2018(1), pp.60-66.

14. Oechslin, F., 2018. Resistance development to bacteriophages occurring during bacteriophage therapy. Viruses, 10(7), p.351.21.

15. LeClerc, J.E., Li, B., Payne, W.L. and Cebula, T.A., 1996. High mutation frequencies among Escherichia coli and Salmonella pathogens. Science, 274(5290), pp.1208-1211.

16. Oliver, A., Cantón, R., Campo, P., Baquero, F. and Blázquez, J., 2000. High frequency of hypermutable Pseudomonas aeruginosa in cystic fibrosis lung infection. Science, 288(5469), pp.1251-1253.

17. Luria, S.E. and Delbrück, M., 1943. Mutations of bacteria from virus sensitivity to virus resistance. Genetics, 28(6), p.491.

18. Wright, R.C., Friman, V.P., Smith, M.C. and Brockhurst, M.A., 2018. Cross-resistance is modular in bacteria-phage interactions. PLoS biology, 16(10), p.e2006057.

19. Rohwer, F., Youle, M., Maughan, H. and Hisakawa, N., 2015. Life in our phage world: a centennial field guide to the Earth's most diverse inhabitants. Wholon.

20. Burrowes, B.H., Molineux, I.J. and Fralick, J.A., 2019. Directed in vitro evolution of therapeutic bacteriophages: The Appelmans protocol. Viruses, 11(3), p.241.

21. Yang, Y., Shen, W., Zhong, Q., Chen, Q., He, X., Baker, J.L., Xiong, K., Jin, X., Wang, J., Hu, F. and Le, S., 2020. Development of a bacteriophage cocktail to constrain the emergence of phage-resistant Pseudomonas aeruginosa. Frontiers in Microbiology, 11, p.327.

22. Rohde, C., Resch, G., Pirnay, J.P., Blasdel, B.G., Debarbieux, L., Gelman, D., Górski, A., Hazan, R., Huys, I., Kakabadze, E. and Lobocka, M., 2018. Expert opinion on three phage therapy related topics: bacterial phage resistance, phage training and prophages in bacterial production strains. Viruses, 10(4), p.178.

23. Laanto, E., Mäkelä, K., Hoikkala, V., Ravantti, J.J. and Sundberg, L.R., 2020. Adapting a Phage to Combat Phage Resistance. Antibiotics, 9(6), p.291. 
24. Woolhouse, M.E., Webster, J.P., Domingo, E., Charlesworth, B. and Levin, B.R., 2002. Biological and biomedical implications of the co-evolution of pathogens and their hosts. Nature genetics, 32(4), pp.569577.

25. Hall, A.R., Scanlan, P.D., Morgan, A.D. and Buckling, A., 2011. Host-parasite coevolutionary arms races give way to fluctuating selection. Ecol. Lett. 14, 635-642.

26. Meyer, J.R., Dobias, D.T., Weitz, J.S., Barrick, J.E., Quick, R.T. and Lenski, R.E., 2012. Repeatability and contingency in the evolution of a key innovation in phage lambda. Science, 335(6067), pp.428-432.

27. Meyer, J.R., Dobias, D.T., Medina, S.J., Servilio, L., Gupta, A. and Lenski, R.E., 2016. Ecological speciation of bacteriophage lambda in allopatry and sympatry. Science, 354(6317), pp.1301-1304.

28. Petrie, K.L., Palmer, N.D., Johnson, D.T., Medina, S.J., Yan, S.J., Li, V., Burmeister, A.R. and Meyer, J.R., 2018. Destabilizing mutations encode nongenetic variation that drives evolutionary innovation. Science, 359(6383), pp.1542-1545.

29. Buckling, A. and Rainey, P.B., 2002. Antagonistic coevolution between a bacterium and a bacteriophage. Proceedings of the Royal Society of London. Series B: Biological Sciences, 269(1494), pp.931-936.

30. Bohannan, B.J. and Lenski, R.E., 2000. Linking genetic change to community evolution: insights from studies of bacteria and bacteriophage. Ecology letters, 3(4), pp.362-377.

31. Chaudhry, W.N., Pleška, M., Shah, N.N., Weiss, H., McCall, I.C., Meyer, J.R., Gupta, A., Guet, C.C. and Levin, B.R., 2018. Leaky resistance and the conditions for the existence of lytic bacteriophage. PLoS biology, 16(8), p.e2005971.

32. Rosche, W.A. and Foster, P.L., 2000. Determining mutation rates in bacterial populations. Methods, 20(1), pp.4-17.

33. Deatherage, D.E. and Barrick, J.E., 2014. Identification of mutations in laboratory-evolved microbes from next-generation sequencing data using breseq. Engineering and analyzing multicellular systems (pp. 165188). Humana Press, New York, NY.

34. Zylicz, M., Ang, D., Liberek, K. and Georgopoulos, C., 1989. Initiation of lambda DNA replication with purified host-and bacteriophage-encoded proteins: the role of the dnaK, dnaJ and grpE heat shock proteins. The EMBO Journal, 8(5), pp.1601-1608.

35. Gupta, A., Peng, S. Leung, C.Y., Borin, J.M., Weitz, J. and Meyer, J.R. Leapfrog dynamics in phagebacteria coevolution revealed by joint analysis of cross-infection phenotypes and whole genome sequencing. bioRxiv doi: 10.1101/2020.10.31.337758

36. Pagnout, C., Sohm, B., Razafitianamaharavo, A., Caillet, C., Offroy, M., Leduc, M., Gendre, H., Jomini, S., Beaussart, A., Bauda, P. and Duval, J.F., 2019. Pleiotropic effects of rfa-gene mutations on Escherichia coli envelope properties. Scientific reports, 9(1), pp.1-16.

37. Roncero, C.E.S.A.R. and Casadaban, M.J., 1992. Genetic analysis of the genes involved in synthesis of the lipopolysaccharide core in Escherichia coli K-12: three operons in the rfa locus. Journal of bacteriology, 174(10), pp.3250-3260.

38. Banzhaf, W., Cheng, B.H., Deb, K., Holekamp, K.E., Lenski, R.E., Ofria, C., Pennock, R.T., Punch, W.F. and Whittaker, D.J., 2020. Evolution in Action: Past, Present and Future.

39. Andrews, B. and Fields, S., 2020. Distinct patterns of mutational sensitivity for $\lambda$ resistance and maltodextrin transport in Escherichia coli LamB. Microbial Genomics, 6(4), p.e000364.

40. Casjens, S., 2003. Prophages and bacterial genomics: what have we learned so far? Molecular microbiology, 49(2), pp.277-300.

41. Juhala, R.J., Ford, M.E., Duda, R.L., Youlton, A., Hatfull, G.F. and Hendrix, R.W., 2000. Genomic sequences of bacteriophages HK97 and HK022: pervasive genetic mosaicism in the lambdoid bacteriophages. Journal of molecular biology, 299(1), pp.27-51.

42. Zhang, H., Fouts, D.E., DePew, J. and Stevens, R.H., 2013. Genetic modifications to temperate Enterococcus faecalis phage $\phi E f 11$ that abolish the establishment of lysogeny and sensitivity to repressor, and increase host range and productivity of lytic infection. Microbiology, 159(Pt 6), p.1023.

43. Monteiro, R., Pires, D.P., Costa, A.R. and Azeredo, J., 2019. Phage therapy: going temperate? Trends in microbiology, 27(4), pp.368-378.

44. Kortright, K.E., Chan, B.K. and Turner, P.E., 2020. High-throughput discovery of phage receptors using transposon insertion sequencing of bacteria. Proceedings of the National Academy of Sciences, 117(31), pp.18670-18679.

45. Yehl, K., Lemire, S., Yang, A.C., Ando, H., Mimee, M., Torres, M.D.T., de la Fuente-Nunez, C. and Lu, T.K., 2019. Engineering phage host-range and suppressing bacterial resistance through phage tail fiber mutagenesis. Cell, 179(2), pp.459-469. 
46. Dunne, M., Rupf, B., Tala, M., Qabrati, X., Ernst, P., Shen, Y., Sumrall, E., Heeb, L., Plückthun, A., Loessner, M.J. and Kilcher, S., 2019. Reprogramming bacteriophage host range through structure-guided design of chimeric receptor binding proteins. Cell reports, 29(5), pp.1336-1350.

47. Roach, D.R., Leung, C.Y., Henry, M., Morello, E., Singh, D., Di Santo, J.P., Weitz, J.S. and Debarbieux, L., 2017. Synergy between the host immune system and bacteriophage is essential for successful phage therapy against an acute respiratory pathogen. Cell host \& microbe, 22(1), pp.38-47.

48. Monferrer, E. and Domingo Calap, P., 2019. Virus-Host Coevolution as a Tool for Controlling Bacterial Resistance to Phage Therapy. Journal of Biotechnology and Biomedicine, 2019, vol. 2, p. 096-104.

49. Peters, T.L., Song, Y., Bryan, D.W., Hudson, L.K. and Denes, T.G., 2020. Mutant and recombinant phages selected from in vitro coevolution conditions overcome phage-resistant Listeria monocytogenes. Applied and Environmental Microbiology.

50. Weissbach, A. and Jacob, F., 1962. Effect of Glucose on the Formation of Bacteriophage ג. Nature, 193(4811), pp.197-198.

51. Howes, W.V., 1965. Effect of glucose on the capacity of Escherichia coli to be infected by a virulent $\lambda$ bacteriophage. Journal of bacteriology, 90(5), pp.1188-1193.

52. Wood, E.J., 1999. At the bench: A laboratory navigator. Edited by Kathy Barker. pp. 460. Cold Spring Harbor Laboratory Press, 1998. \$45. ISBN 0-87969-523-4. Biochemical Education, 27(2), pp.124-125.

53. R Development Core Team (2019) R: a language and environment for statistical computing. R Foundation for Statistical Computing, Vienna http://www.R-project.org. Accessed Sept 2019.

54. Burmeister, A.R., Lenski, R.E. and Meyer, J.R., 2016. Host coevolution alters the adaptive landscape of a virus. Proceedings of the Royal Society B: Biological Sciences, 283(1839), p.20161528.

55. Shao, Y. and Wang, N., 2008. Bacteriophage adsorption rate and optimal lysis time. Genetics, 180(1), pp.471-482. 